\title{
Ophthalmic infections in children presenting to Angkor Hospital for Children, Siem Reap, Cambodia
}

\author{
Phara Khauv' ${ }^{1}$, Paul Turner ${ }^{2,3,4^{*}}$, Channy Soeng ${ }^{1}$, Sona Soeng ${ }^{1}$, Catrin E Moore ${ }^{3,4}$, Rachel Bousfield ${ }^{3,4}$, \\ Nicole Stoesser ${ }^{3,4}$, Kate Emary ${ }^{3,4}$, Duy Pham Thanh ${ }^{5}$, Stephen Baker ${ }^{4,5,6}$, Vu thi Ty Hang ${ }^{5}$, H Rogier van Doorn ${ }^{4,5}$, \\ Nicholas PJ Day 2,3,4 and Christopher M Parry ${ }^{3,4}$
}

\begin{abstract}
Background: Ophthalmic infections cause significant morbidity in Cambodian children but aetiologic data are scarce. We investigated the causes of acute eye infections in 54 children presenting to the ophthalmology clinic at Angkor Hospital for Children, Siem Reap between March and October 2012.

Findings: The median age at presentation was 3.6 years (range 6 days -16.0 years). Forty two patients (77.8\%) were classified as having an external eye infection, ten (18.5\%) as ophthalmia neonatorum, and two (3.7\%) as intra-ocular infection. Organisms were identified in all ophthalmia neonatorum patients and $85.7 \%$ of patients with an external eye infection. Pathogens were not detected in either of the intra-ocular infection patients. Most commonly isolated bacteria were Staphylococcus aureus (23 isolates), coagulase-negative staphylococci (13), coliforms (7), Haemophilus influenzae/parainfluenzae (6), Streptococcus pneumoniae (4), and Neisseria gonorrhoeae (2). Chlamydia trachomatis DNA was detected in $60 \%$ of swabs taken from ophthalmia neonatorum cases.

Conclusions: This small study demonstrates the wide range of pathogens associated with common eye infections in Cambodian children. The inclusion of molecular assays improved the spectrum of detectable pathogens, most notably in neonates.
\end{abstract}

Keywords: Paediatric, Ophthalmic, Infection, Chlamydia

\section{Findings}

Ophthalmic infections are a major cause of acute and chronic morbidity in Cambodian children, and one of the commonest causes of visual impairment in this population [1-3]. However, there are limited data on the spectrum of microbial organisms causing these infections in Cambodia, since diagnostic laboratory facilities and ophthalmology services are scare, with an estimate of less than twenty trained ophthalmologists practising in the entire country (http://www.hollows.org.au/ourwork/where-we-work-country/cambodia). The aim of this study was to make a preliminary assessment of the

\footnotetext{
* Correspondence: pault@tropmedres.ac

${ }^{2}$ Cambodia-Oxford Medical Research Unit, Angkor Hospital for Children, Siem Reap, Cambodia

${ }^{3}$ Mahidol-Oxford Tropical Medicine Research Unit, Faculty of Tropical

Medicine, Mahidol University, Bangkok, Thailand

Full list of author information is available at the end of the article
}

causes of infections in children presenting to the ophthalmic service at Angkor Hospital for Children (AHC) in Siem Reap to aid empirical prescribing practice, inform public health interventions and establish a surveillance baseline for the monitoring of disease trends. The study protocol was reviewed and approved by the Institutional Review Board at Angkor Hospital for Children, Cambodia and the Oxford Tropical Ethics Committee, UK (ref 46-11).

Between $1^{\text {st }}$ March and $31^{\text {st }}$ October 2012, children presenting to the AHC eye clinic with clinical evidence of ophthalmic infection were invited to participate in the study and written consent obtained from the attending parent or caregiver. Swabs, corneal scrapings, or intraocular specimens were submitted for microscopy and bacterial culture, according to the clinical diagnosis. Specimens were transported to the onsite microbiology 
immediately after collection and were cultured on a variety of culture media (all specimens: $5 \%$ sheep blood agar, chocolate agar, MacConkey agar, Sabouraud agar, fastidious anaerobe agar (Oxoid, Basingstoke, UK; prepared in house); if a specimen from a neonate: additional selective plate for isolation of Neisseria gonorrhoeae; if corneal scrape or intra-ocular pus specimen: additional culture in thioglycollate broth with subculture to fastidious anaerobe agar after 48 hours) and incubated for up to 48 hours aerobically, anaerobically, or in $5-10 \%$ $\mathrm{CO}_{2}$ as appropriate. Cultured organisms were identified by colony morphology, Gram stain characteristics, and standard microbiologic techniques [4]. Antimicrobial susceptibilities were performed by disk diffusion according to current Clinical Laboratory Standards Institute (CLSI) guidelines [5]. Benzyl penicillin and ceftriaxone minimum inhibitory concentrations (MIC) were determined by Etest (bioMerieux, Marcy L'Etoile, France) for Streptococcus pneumoniae isolates with a $1 \mu \mathrm{g}$ oxacillin disc zone diameter of $<20 \mathrm{~mm}$, and interpreted using the CLSI guidelines. In addition to bacterial culture, an aliquot of each specimen was stored at $-80^{\circ} \mathrm{C}$ in $200 \mu \mathrm{L}$ sterile phosphate buffered saline (PBS) for molecular analysis. Nucleic acids were extracted from the stored specimen aliquots using the NucliSENS easyMag system (bioMerieux, Marcy L'Etoile, France) and Chlamydia trachomatis, adenovirus, and herpes simplex virus (HSV) 1 and 2 infections were detected by real-time PCR assays, as previously described [6-8].

Fifty four patients were enrolled in the study. The median age at presentation was 3.6 years (interquartile range $0.3-11.1$ years; range 6 days -16.0 years). Thirty patients $(30 / 54 ; 55.6 \%)$ were male. Forty two patients $(42 / 54 ; 77.8 \%)$ were classified as having an external eye infection (conjunctivitis (11), corneal ulcer (11), dacrocystitis (7), stye (7), eyelid abscess (6)), ten (18.5\%) as ophthalmia neonatorum, and two (3.7\%) as intra-ocular infection. At least one bacterial or viral species was detected from specimens submitted for 46 patients (46/54; 85.2\%; Table 1): more than one organism was detected in a third of specimens $(17 / 54 ; 31.5 \%$; Table 2$)$. Potentially pathogenic organisms were identified in all $(10 / 10)$ ophthalmia neonatorum patients and $85.7 \%(36 / 42)$ of patients with an external eye infection. There was no growth from specimens in both of the intra-ocular infection patients. Antimicrobial susceptibility test results for

Table 1 Culture and PCR results by category of infection

\begin{tabular}{|c|c|c|c|c|}
\hline Organism & $\begin{array}{l}\text { Intra-ocular infection } \\
\mathrm{N} \text { (column \%) }\end{array}$ & $\begin{array}{l}\text { External eye infection } \\
\mathrm{N} \text { (column \%) }\end{array}$ & $\begin{array}{l}\text { Ophthalmia neonatorum } \\
\mathrm{N} \text { (column \%) }\end{array}$ & $\begin{array}{l}\text { Total } \\
\mathrm{N} \text { (column \%) }\end{array}$ \\
\hline Number (row \%) & $2(3.7)$ & $42(77.8)$ & $10(18.5)$ & 54 \\
\hline \multicolumn{5}{|l|}{ Culture-based detection } \\
\hline Staphylococcus aureus & $0(0)$ & $19(45.2)$ & $4(40.0)$ & $23(42.6)$ \\
\hline CONS $^{1}$ & $0(0)$ & $9(21.4)$ & $4(40.0)$ & $13(24.1)$ \\
\hline Gram positive bacilli & $0(0)$ & $3(7.1)$ & $4(40.0)$ & $7(13.0)$ \\
\hline Enterobacteriaceae $^{2}$ & $0(0)$ & $4(9.5)$ & $3(30.0)$ & $5(9.3)^{3}$ \\
\hline Haemophilus influenzae & $0(0)$ & $4(9.5)$ & $0(0)$ & $4(7.4)$ \\
\hline Streptococcus pneumoniae & $0(0)$ & $4(9.5)$ & $0(0)$ & $4(7.4)$ \\
\hline Haemophilus parainfluenzae & $0(0)$ & $1(2.4)$ & $1(10.0)$ & $2(3.7)$ \\
\hline Neisseria gonorrhoeae & $0(0)$ & $0(0)$ & $2(20.0)$ & $2(3.7)$ \\
\hline Pseudomonas aeruginosa & $0(0)$ & $2(4.8)$ & $0(0)$ & $2(3.7)$ \\
\hline Burkholderia pseudomallei & $0(0)$ & $1(2.4)$ & $0(0)$ & $1(1.9)$ \\
\hline Candida sp. & $0(0)$ & $1(2.4)$ & $0(0)$ & $1(1.9)$ \\
\hline Enterococcus sp. & $0(0)$ & $0(0)$ & $1(10.0)$ & $1(1.9)$ \\
\hline Moraxella catarrhalis & $0(0)$ & $0(0)$ & $1(10.0)$ & $1(1.9)$ \\
\hline Streptococcus bovis & $0(0)$ & $0(0)$ & $1(10.0)$ & $1(1.9)$ \\
\hline \multicolumn{5}{|l|}{ PCR-based detection } \\
\hline Adenoviruses (AdV) & $0(0)$ & $0(0)$ & $0(0)$ & $0(0)$ \\
\hline Herpes Simplex viruses (HSV) & $0(0)$ & $1(2.4)$ & $0(0)$ & $1(1.9)$ \\
\hline Chlamydia trachomatis & $0(0)$ & $1(2.4)$ & $6(60.0)$ & $6(11.1)$ \\
\hline
\end{tabular}

${ }^{1}$ Coagulase-negative staphylococci.

${ }^{2}$ Coliforms: Klebsiella pneumoniae (2), Enterobacter cloacae (1), Escherichia coli (1), Morganella morganii (1), Pantoea sp. (1), Serratia plymuthica (1). ${ }^{3} \mathrm{Number}(\%)$ of specimens from which at least one member of the Enterobacteriaceae was isolated (there were two swabs with two species of Enterobacteriaceae isolated). 
Table 2 Culture details of the seventeen polymicrobial infections

\begin{tabular}{|c|c|c|c|}
\hline Diagnosis & Gram positive & Gram negative & Other organisms \\
\hline \multirow[t]{2}{*}{ Conjunctivitis } & Staphylococcus aureus & Haemophilus influenzae & Chlamydia trachomatis \\
\hline & Streptococcus pneumoniae & & \\
\hline \multirow[t]{2}{*}{ Conjunctivitis } & CoNS $^{1}$ & Pantoea sp. & \\
\hline & & Serratia plymuthica & \\
\hline Conjunctivitis & S. aureus & Pseudomonas aeruginosa & \\
\hline Conjunctivitis & S. aureus & H. parainfluenzae & \\
\hline Conjunctivitis & CoNS & H. influenzae & \\
\hline \multirow[t]{2}{*}{ Conjunctivitis } & CoNS & H. influenzae & \\
\hline & Gram positive bacillus & & \\
\hline \multirow[t]{2}{*}{ Conjunctivitis } & S. pneumoniae & & \\
\hline & CoNS & & \\
\hline \multirow[t]{2}{*}{ Corneal ulcer } & & Enterobacter cloacae & \\
\hline & & Morganella morganii & \\
\hline \multirow[t]{2}{*}{ Dacrocystitis } & CoNS & & \\
\hline & Gram positive bacillus & & \\
\hline Stye & S. aureus & & Candida sp. \\
\hline Ophthalmia neonatorum & S. aureus & Neisseria gonorrhoeae & C. trachomatis \\
\hline Ophthalmia neonatorum & S. aureus & Moraxella catarrhalis & \\
\hline \multirow[t]{2}{*}{ Ophthalmia neonatorum } & Enterococcus sp. & H. parainfluenzae & \\
\hline & S. aureus & Klebsiella pneumoniae & \\
\hline \multirow[t]{2}{*}{ Ophthalmia neonatorum } & S. aureus & K. pneumoniae & C. trachomatis \\
\hline & CoNS & & \\
\hline \multirow[t]{2}{*}{ Ophthalmia neonatorum } & Streptococcus bovis & & C. trachomatis \\
\hline & Gram positive bacillus & & \\
\hline Ophthalmia neonatorum & CoNS & N. gonorrhoeae & \\
\hline \multirow[t]{2}{*}{ Ophthalmia neonatorum } & CoNS & & C. trachomatis \\
\hline & Gram positive bacillus & & \\
\hline
\end{tabular}

${ }^{1}$ Coagulase-negative staphylococci.

commonly cultured organisms are shown in Table 3. Chlamydia trachomatis DNA was detected in swabs from seven patients $(7 / 54 ; 13.0 \%)$ : six were ophthalmia neonatorum presentations and one of these was a coinfection with Neisseria gonorrhoeae. HSV DNA was

Table 3 Antimicrobial susceptibility results of commonly cultured organisms

\begin{tabular}{lllllllll}
\hline Organism & \multicolumn{9}{l}{ Drug (\% susceptible) } \\
\cline { 2 - 9 } & AMP $^{\mathbf{1}}$ & $\mathbf{A M C}^{\mathbf{1}}$ & $\mathbf{O X}^{\mathbf{1}}$ & $\mathbf{P}^{\mathbf{1}}$ & $\mathbf{C R O}^{\mathbf{1}}$ & $\mathbf{C I P}^{\mathbf{1}}$ & $\mathbf{S X T}^{\mathbf{1}}$ & $\mathbf{C N}^{\mathbf{1}}$ \\
\hline S. aureus & $\mathrm{ND}^{2}$ & $\mathrm{ND}$ & 95.7 & 4.4 & $\mathrm{ND}$ & 100 & 95.7 & 100 \\
S. pneumoniae & $\mathrm{ND}$ & $\mathrm{ND}$ & $\mathrm{ND}$ & 100 & 100 & $\mathrm{ND}$ & 25.0 & $\mathrm{ND}$ \\
H. influenzae & 83.3 & 100 & $\mathrm{ND}$ & $\mathrm{ND}$ & 100 & 100 & 40.0 & $\mathrm{ND}$ \\
N. gonorrhoeae & $\mathrm{ND}$ & $\mathrm{ND}$ & $\mathrm{ND}$ & 0 & 100 & 0 & $\mathrm{ND}$ & $\mathrm{ND}$ \\
Enterobacteriaceae & 42.9 & 85.7 & $\mathrm{ND}$ & $\mathrm{ND}$ & 83.3 & 85.7 & 85.7 & 85.7 \\
\hline
\end{tabular}

${ }^{1} \mathrm{AMP}$ - ampicillin; AMC - co-amoxiclav; OX - oxacillin; $\mathrm{P}$ - penicillin; CRO - ceftriaxone; CIP - ciprofloxacin; SXT - co-trimoxazole; CN - gentamicin. ${ }^{2}$ Not done/not applicable. detected from a swab in one child presenting with corneal ulceration. Adenovirus DNA was not detected in any specimens.

This study, although small, demonstrates the wide range of pathogens responsible for common eye infections in Cambodian children. These organisms are generally similar to those found in reports from other populations $[9,10]$. Variations between study results may reflect age distribution of cases, contact lens use (a risk factor for keratitis), and host factors such as malnutrition and immunisation status [11-13]. However, the absence of confirmed adenovirus or fungal infection was surprising and perhaps attributable to the small sample size, the limited duration of the study (which have resulted in a missed seasonal adenovirus outbreak), specimen factors (i.e. most specimens were swabs), and the short incubation period for fungal culture plates (although fungal elements were not seen on microscopy 
in any of the specimens). Larger studies in this population maybe warranted to validate the current findings. The isolation of Burkholderia pseudomallei, the cause of melioidosis, from an eyelid abscess is noteworthy and demonstrates the diversity of presentation of infections with this organism [14]. Staphylococcus aureus was the most frequently cultured organism and the majority $(22 / 23 ; 95.7 \%)$ of isolates were meticillin sensitive, although with the emergence of community-acquired meticillinresistant S. aureus (MRSA) infections in Cambodia this may change with time [15]. Isolates of Neisseria gonorrhoeae were penicillin and fluoroquinolone resistant but susceptible to ceftriaxone, which is similar to the findings of other eye infection studies from Southeast Asia [16,17]. The addition of molecular testing increased the spectrum of detectable pathogens, most notably in neonates where $60 \%$ of infections were associated with detection of Chlamydia trachomatis DNA. Maternal screening for sexually transmitted infections (STI) in pregnancy and tetracycline eye ointment administration for newborns are not routine practice in Cambodia but may be desirable given the frequent isolation of STI-related organisms from ophthalmia neonatorum cases. These data will serve as a useful baseline for further studies of these important infections in Cambodia.

\section{Competing interests}

The authors declare that they have no competing interests.

\section{Authors' contributions \\ $P K, C S, N S, C M, K E, S B, R D, N D$, and CP conceived the study. PK, CS, and RB collected the clinical specimens and data. SS, CM, DT, VH, SB, and RD performed the laboratory work. PT and PK analysed the data. PT and CP wrote the paper. All authors contributed to revisions of the manuscript and approved the final version.}

\section{Acknowledgements}

This work was supported by the Wellcome Trust of the United Kingdom and the Li Ka Shing Foundation - University of Oxford Global Health Programme. Stephen Baker is a Sir Henry Dale Fellow, jointly funded by the Wellcome Trust and the Royal Society (100087/Z/12/Z).

\footnotetext{
Author details

${ }^{1}$ Angkor Hospital for Children, Siem Reap, Cambodia. ${ }^{2}$ Cambodia-Oxford Medical Research Unit, Angkor Hospital for Children, Siem Reap, Cambodia. ${ }^{3}$ Mahidol-Oxford Tropical Medicine Research Unit, Faculty of Tropical Medicine, Mahidol University, Bangkok, Thailand. ${ }^{4}$ Centre for Tropical Medicine, Nuffield Department of Medicine, University of Oxford, Oxford, UK. ${ }^{5}$ The Hospital for Tropical Diseases, Wellcome Trust Major Overseas Programme, Oxford University Clinical Research Unit, Ho Chi Minh City, Vietnam. ${ }^{6}$ The London School of Hygiene and Tropical Medicine, London, United Kingdom.
}

Received: 20 December 2013 Accepted: 21 October 2014 Published: 5 November 2014

\section{References}

1. Thomson I: A clinic based survey of blindness and eye disease in Cambodia. Br J Ophthalmol 1997, 81(7):578-580.

2. Jackson H, Foster A: Causes of blindness in northwest Cambodia. Ophthalmic Epidemiol 1997, 4(1):27-32.
3. Rutzen AR, Ellish NJ, Schwab L, Graham PJ, Pizzarello LD, Hemady RK, Maldonado MJ: Blindness and eye disease in Cambodia. Ophthalmic Epidemiol 2007, 14(6):360-366.

4. Dumler JS, Funke G, Janda JM, Nachamkin I, Versalovic J: Bacteriology. In Manual of Clinical Microbiology. Volume 1. 9th edition. Edited by Murray PR, Baron EJ, Jorgensen JH, Landry ML, Pfaller MA. Washington DC: ASM Press; 2007:272-1074.

5. CLSI: Performance Standards for Antimicrobial Susceptibility Testing; Seventeenth Informational Supplement. CLSI document M100-S23. Clinical and Laboratory Standards Institute: Wayne, PA; 2013.

6. Jalal H, Stephen H, Curran MD, Burton J, Bradley M, Carne C: Development and validation of a rotor-gene real-time PCR assay for detection, identification, and quantification of Chlamydia trachomatis in a single reaction. J Clin Microbiol 2006, 44(1):206-213.

7. Jansen RR, Schinkel J, Koekkoek S, Pajkrt D, Beld M, De Jong MD, Molenkamp $R$ : Development and evaluation of a four-tube real time multiplex PCR assay covering fourteen respiratory viruses, and comparison to its corresponding single target counterparts. J Clin Virol 2011, 51(3):179-185.

8. Van Doornum GJ, Guldemeester J, Osterhaus AD, Niesters HG: Diagnosing herpesvirus infections by real-time amplification and rapid culture. J Clin Microbiol 2003, 41(2):576-580.

9. Sergiwa A, Pratt BC, Eren E, Sunona TC, Hart CA: Ophthalmia neonatorum in Bangkok: the significance of Chlamydia trachomatis. Ann Trop Paediatr 1993, 13(3):233-236.

10. Shah A, Sachdev A, Coggon D, Hossain P: Geographic variations in microbial keratitis: an analysis of the peer-reviewed literature. Br J Ophthalmol 2011, 95(6):762-767.

11. Young AL, Leung KS, Tsim N, Hui M, Jhanji V: Risk factors, microbiological profile, and treatment outcomes of pediatric microbial keratitis in a tertiary care hospital in Hong Kong. Am J Ophthalmol 2013, 156(5):1040-1044. e1042.

12. Lee YS, Tan HY, Yeh LK, Lin HC, Ma DH, Chen HC, Chen SY, Chen PY, Hsiao CH: Pediatric microbial keratitis in Taiwan: clinical and microbiological profiles, 1998-2002 versus 2008-2012. Am J Ophthalmol 2014, 157(5):1090-1096.

13. Jhanji V, Naithani P, Lamoureux E, Agarwal T, Sharma N, Vajpayee RB: Immunization and nutritional profile of cases with atraumatic microbial keratitis in preschool age group. Am J Ophthalmo/ 2011, 151(6):1035-1040. e1032.

14. Pagnarith Y, Kumar V, Thaipadungpanit J, Wuthiekanun V, Amornchai P, Sin L, Day NP, Peacock SJ: Emergence of pediatric melioidosis in Siem Reap, Cambodia. Am J Trop Med Hyg 2010, 82(6):1106-1112.

15. Chheng K, Tarquinio S, Wuthiekanun V, Sin L, Thaipadungpanit J, Amornchai P, Chanpheaktra N, Tumapa S, Putchhat H, Day NP, Peacock SJ: Emergence of community-associated methicillin-resistant Staphylococcus aureus associated with pediatric infection in Cambodia. PLoS One 2009, 4(8):e6630.

16. Vernel-Pauillac F, Ratsima EH, Guillard B, Goursaud R, Lethezer C, Hem S, Merien F, Goarant C: Correlation between antibiotic susceptibilities and genotypes in Neisseria gonorrhoeae from different geographical origins: determinants monitoring by real-time PCR as a complementary tool for surveillance. Sex Transm Infect 2010, 86(2):106-111.

17. Olsen B, Pham TL, Golparian D, Johansson E, Tran HK, Unemo M: Antimicrobial susceptibility and genetic characteristics of Neisseria gonorrhoeae isolates from Vietnam, 2011. BMC Infect Dis 2013, 13:40.

doi:10.1186/1756-0500-7-784

Cite this article as: Khauv et al:: Ophthalmic infections in children presenting to Angkor Hospital for Children, Siem Reap, Cambodia. BMC Research Notes 2014 7:784. 